\title{
Characterization and comparison of the temporal dynamics of ruminal bacterial microbiota colonizing rice straw and alfalfa hay within ruminants
}

\author{
Junhua Liu, Mengling Zhang, Chunxu Xue, Weiyun Zhu, and Shengyong Mao' \\ Jiangsu Key Laboratory of Gastrointestinal Nutrition and Animal Health, College of Animal Science and Technology, Nanjing Agricultural \\ University, Nanjing 210095, China
}

\begin{abstract}
Three ruminally cannulated Holstein cows were used to characterize the dynamics of bacterial colonization of rice straw and alfalfa hay and to assess the differences in the composition and inferred gene function of the colonized microbiota between these 2 forages. Nonincubated $(0 \mathrm{~h})$ rice straw and alfalfa hay samples and residues in nylon bags incubated for $0.5,2,6,16$, and $48 \mathrm{~h}$ were analyzed for dry matter and were used for DNA extraction and MiSeq (Illumina Inc., San Diego, $\mathrm{CA}$ ) sequencing of the $16 \mathrm{~S}$ rRNA gene. The microbial communities that colonized the air-dried and nonincubated $(0 \mathrm{~h})$ rice straw and alfalfa hay were both dominated by members of the Proteobacteria (contributing toward $70.47 \%$ of the $16 \mathrm{~S}$ RNA reads generated). In situ incubation of the 2 forages revealed major shifts in the community composition: Proteobacteria were replaced within $30 \mathrm{~min}$ by members belonging to the Bacteroidetes and Firmicutes, contributing toward 51.9 and $36.6 \%$ of the $16 \mathrm{~S}$ rRNA reads generated, respectively. A second significant shift was observed after $6 \mathrm{~h}$ of rumen incubation, when members of the Spirochaetes and Fibrobacteria phyla became abundant in the forageadherent community. During the first 30 min of rumen incubation, $\sim 20.7$ and $36.1 \%$ of the rice straw and alfalfa hay, respectively, were degraded, whereas little biomass degradation occurred between $30 \mathrm{~min}$ and $2 \mathrm{~h}$ after the rice straw or alfalfa hay was placed in the rumen. Significant differences were noted in attached bacterial community structure between the 2 forage groups, and the abundances of dominant genera Anaeroplasma, Butyrivibrio, Fibrobacter, and Prevotella were affected by the forage types. Real-time PCR results showed that the $16 \mathrm{~S}$ rRNA copies of total bacteria attached to these 2 forages were affected by the forage types and incubation time, and higher numbers of attached bacterial $16 \mathrm{~S}$
\end{abstract}

Received May 4, 2016.

Accepted August 13, 2016.

${ }^{1}$ Corresponding author: maoshengyong@163.com
rRNA were observed in the alfalfa hay samples than in the rice straw from 0.5 to $16 \mathrm{~h}$ of incubation. The metagenomes predicted by phylogenetic investigation of communities by reconstruction of unobserved states (PICRUSt) revealed that the forage types significantly affected 21 metabolic pathways identified in the Kyoto Encyclopedia of Genes and Genomes, and 33 were significantly changed over time. Collectively, our results reveal a difference in the dynamics of bacterial colonization and the inferred gene function of microbiota associated with rice straw and alfalfa hay within the rumen. These findings are of great importance for the targeted improvement of forage nutrient use efficiency in ruminants.

Key words: rumen, rice straw, alfalfa hay, microbiome

\section{INTRODUCTION}

Ruminant animals and ruminal microorganisms have evolved together for millions of years, resulting in a rumen microbial population consisting of a highly diverse collection of obligately anaerobic microorganisms, including fungi, protozoa, bacteria, and archaea (Russell and Rychlik, 2001). Within this microbiome, bacteria are the dominant domain and make the greatest contribution to digestion and conversion of feeds to volatile fatty acids and microbial proteins (Kim et al., 2011). A previous study showed that bacterial attachment is instrumental in the process of microbial degradation of dietary plant material (McAllister et al., 1994), and particle-associated bacteria account for 70 to $80 \%$ of rumen microbial matter (Craig et al., 1987). Therefore, studies on the mode of bacterial attachment and the subsequent digestion of the plant biomass are essential for improving ruminant nutrient use efficiency.

Over the last decade, many experiments have demonstrated that rumen microorganisms rapidly associate with and colonize recently ingested feed particles (Edwards et al., 2007; Huws et al., 2013, 2016; Piao et al., 2014, 2015), and these studies have revealed that the composition of colonizing microbial communities is affected by the incubation time (Bowman and Firkins, 
1993; Huws et al., 2013, 2016; Piao et al., 2014, 2015). Forage type is recognized as an important factor that affects the rumination time, rumen fermentation, and performance of the ruminant (Welch and Smith, 1969; Brask et al., 2013; Ding et al., 2015). One forage type, rice straw, is abundant and inexpensive, but is typically considered low-quality roughage. A second type, alfalfa, is well known for its high quality and is used worldwide as an important dietary forage in ruminant production; nevertheless, rice straw is the predominant forage source for ruminants in the tropical zones of the world. These 2 forages are known to differ in the digestibility of their DM in the rumen (Yang et al., 2011), and these differences could be attributed to differences in the physical structure and chemical characteristics between the 2 types of forage. However, the difference in the composition of degrading and colonizing ruminal bacterial communities of the 2 forages may also be an important factor.

In the current study, we hypothesized that forages with different quality will affect the composition of the microbiota that colonize and degrade the forage within the rumen, which will likely be accompanied by a difference in community function. Therefore, the primary objective of this study was to compare the effects of forage sources (rice straw vs. alfalfa hay) on the composition, structure, and inferred function of ruminal particle-associated microbiota in dairy cows. An additional aim was to investigate the temporal changes in the bacterial communities that colonize and degrade rice straw and alfalfa hay in the rumen.

\section{MATERIALS AND METHODS}

\section{Animals and Sample Collection}

Three mature, rumen-cannulated, nonlactating Holstein cows (average BW: $502 \pm 25 \mathrm{~kg}$ ) were used in this study. Cows were individually housed in 3 stalls in a barn with good ventilation and were fed $15 \mathrm{~kg}$ of DM (75\% Chinese wild grass hay, 25\% corn-based diet) per animal per day. The cattle were fed at 0700 and $1730 \mathrm{~h}$, with one-half of the allowed daily ration given at each feeding. The cattle had free access to drinking water. All animal care procedures were approved by the Institutional Animal Care and Use Committee of Nanjing Agricultural University.

Air-dried rice straw and alfalfa hay were ground into 2-mm pieces using a Wiley mill, and the ground samples were weighed into individual in situ nylon bags $(10 \times 20 \mathrm{~cm}$; pore size $=50 \mu \mathrm{m})$. Three bags containing $2 \mathrm{~g}$ of forage for each of the 2 forage treatments and 1 blank bag (i.e., a total of 7 bags for each of the 5 time periods, for a total of 35 bags per cow) were placed into the rumen of each cow and removed after 0.5, 2, 6,16 , and $48 \mathrm{~h}$. After removal from the rumen, 4 bags containing forages ( 2 for rice straw and 2 for alfalfa hay) and the blank bag were rinsed and manipulated in cold water until the water ran clear, then squeezed by hand to remove excess water and used for nutritional ingredient degradation analysis. The remaining 2 bags (one filled with rice straw and one with alfalfa for each cow) were washed with sterile saline to remove loosely attached microbes and then immediately frozen on dry ice and transported to the laboratory for storage at $-80^{\circ} \mathrm{C}$ until DNA extraction.

\section{Chemical Analysis}

Relative biomass degradation during rumen incubation was determined by DM, CP, NDF, and ADF analysis. Dry matter and CP were determined according to AOAC methods (AOAC, 1990), and NDF and ADF were determined using the procedures of Larter (1992).

\section{DNA Extraction and Quantitative Real-Time PCR Analysis}

Total microbial genomic DNA was extracted from $200 \mathrm{mg}$ of the nonincubated control sample and from each rumen-incubated rice straw and alfalfa sample. The DNA was extracted by a bead-beating method using a mini-bead beater (Biospec Products, Bartlesville, OK), followed by phenol-chloroform extraction, as described by Mao et al. (2012). The concentration and purity of DNA were determined using a NanoDrop Spectrophotometer (Thermo Scientific, Wilmington, $\mathrm{DE}$ ), and DNA samples were stored at $-80^{\circ} \mathrm{C}$ until further processing.

Total attached bacterial 16S rDNA was quantified by real-time PCR. Primer pairs for the total bacteria were bacF (5'-CCATTGTAGCACGTGTGTAGCC-3') and total bacR (5'-CGGCAACGAGCGCAACCC-3'), as reported by Hook et al. (2011). The PCR amplifications were performed in triplicate with SYBR Green PCR Mastermix (Applied Biosystems, Foster City, CA) using a CFX96 Real-Time PCR Detection System (Bio-Rad Laboratories, Hertfordshire, UK). The total volume of reaction solution $(20 \mu \mathrm{L})$ contained $0.5 \mathrm{ng}$ of DNA, $10 \mu \mathrm{L}$ of Fast SYBR Green Master Mix (Applied Biosystems), $0.4 \mu \mathrm{L}$ of each primer $(5 \mu M), 6.8 \mu \mathrm{L}$ of nuclease-free water, $0.4 \mu \mathrm{L}$ of ROX Reference Dye $(50 \times)$, and $2 \mu \mathrm{L}$ of the template, as described previously (Mao et al., 2015). Amplification was carried out using the following program: $95^{\circ} \mathrm{C}$ for $30 \mathrm{~s}$ for the initial denaturation and then 40 cycles of $95^{\circ} \mathrm{C}$ for $5 \mathrm{~s}$ followed by annealing/extension for $30 \mathrm{~s}$ at $60^{\circ} \mathrm{C}$. A standard 
curve was constructed using serial dilutions of plasmid DNA containing the 16S rRNA gene sequence of Streptococcus bovis. The real-time PCR efficiency ranged between 90 and 103\%; negative controls without the DNA template were run with every assay to assess the overall specificity.

\section{PCR Amplification, MiSeq Sequencing, and Sequencing Data Processing}

Amplification and sequencing were performed as described elsewhere (Mao et al., 2015). Briefly, the V3V4 regions of bacterial $16 \mathrm{~S}$ rRNA genes were amplified using primers $338 \mathrm{~F}$ (5'-barcode-ACTCCTRCGGGAGGCAGCAG)-3' and 806R (5'-GGACTACCVGGGTATCTAAT- $3^{\prime}$ ), where the barcode is an 8-base sequence unique to each sample. Amplicons were purified and then pooled in equimolar amounts and paired-end sequenced $(2 \times 250)$ on an Illumina MiSeq platform (Illumina Inc., San Diego, CA) according to standard protocols. For data analysis, raw Illumina fastq files were demultiplexed, quality filtered, and analyzed using the Quantitative Insights into Microbial Ecology (QIIME, v.1.8.0), as described by Caporaso et al. (2010b) with the following criteria, as described by Mao et al. (2015): operational taxonomic units (OTU) were clustered with a $97 \%$ similarity cutoff using UPARSE (Edgar, 2013), and chimeric sequences were identified and removed using UCHIME (Edgar et al., 2011). The most abundant sequences within each OTU (representative sequences) were aligned to the Greengenes database using PyNAST (Caporaso et al., 2010a) with the default parameters set by QIIME. Taxonomy was assigned to representative sequences using the Ribosomal Database Project (RDP) classifier (Wang et al., 2007) with a confidence value of 0.8 against the Greengenes $16 \mathrm{~S}$ rRNA gene data set (v.13.8) (DeSantis et al., 2006). A phylogenetic tree was constructed within QIIME using FastTree. Rarefaction curves, $\alpha$ diversity, and $\beta$ diversity calculations were also performed using QIIME. Principal coordinates analysis (PCoA) was used to compare groups of samples based on unweighted UniFrac distance metrics (Lozupone and Knight, 2005), and an unweighted distance-based analysis of molecular variance (AMOVA) was conducted to assess significant differences among samples using the Mothur v.1.3.0 program (Schloss et al., 2009). The similarity between the microbial communities associated with the 2 forages was analyzed through a hierarchical cluster analysis using the average-neighbor method. This analysis was conducted using the function hclust in the $\mathrm{R}$ stats package (R Project 3.0.2; http://cran.r-project. $\operatorname{org} /)$.

\section{Functional Gene Prediction}

Phylogenetic investigation of communities by reconstruction of unobserved states (PICRUSt) is a bioinformatics tool that uses $16 \mathrm{~S}$ ribosomal DNA sequences to predict the functional gene content of microorganisms (Langille et al., 2013). In the present study, we used PICRUSt to obtain an overview of the genomic and metabolic features represented by the adherent bacterial communities in our samples. We associated OTUs with known bacterial genomes precalculated in PICRUSt, by first picking closed OTUs against the Greengenes 16S rRNA gene database (13.5) using QIIME 1.7.0 (Caporaso et al., 2010b). The resulting OTU table was then normalized using the script normalize_by_copy_number.py and used for metagenome inference of Kyoto Encyclopedia of Genes and Genomes (KEGG) Orthologs using PICRUSt. The difference in the predicted molecular functions of the bacterial communities attached to the rice straw and alfalfa hay for the different incubation times was determined by partial least squares discriminant analysis (PLS-DA) using the SIMCA-P (11.5) software package (Umetrics, Umeå, Sweden).

\section{Statistical Analysis}

We related changes in total microbial composition to the chemistry of forage using redundancy analysis (RDA), as implemented in the Canoco 5.0 software package (Microcomputer Power, Ithaca, NY; Ter Braak and Smilauer, 2012). Different chemical composition was introduced as environmental (explanatory) variables. The relative contributions of the top 30 genus-level phylogenetic groups were used as response variables. Redundancy analysis was performed focusing on intersample correlation, and the Monte Carlo Permutation test was applied to decide whether chemical composition had any statistically significant influence on the microbial composition. Chemical ingredients were considered to have significant effects on microbial composition for $P$ values $<0.05$.

The DM loss, real-time PCR data, bacterial prevalence, and relative abundance values of the KEGG pathways were analyzed using general linear model (GLM) of SPSS (SPSS v.16, SPSS Inc., Chicago, IL) according to the following equation: $Y_{i j k}=\mu+G_{i}+$ $T_{j}+G T_{i j}+e_{i j k}$, where $Y_{i j k}$ was the observation, $\mu$ was the overall mean, $G_{i}$ was the forage effect $(i=2), T_{j}$ was the incubation time effect $(j=5), G T_{i j}$ was the interaction between the forage types and incubation time, and $e_{i j k}$ was the residual error. All $P$-values from the GLM tests of relative abundance of bacterial taxa 
were corrected for a false discovery rate (FDR) of 0.05 using the Benjamini-Hochberg method (Benjamini and Hochberg, 2000), and FDR-corrected $P$-values below 0.05 (FDR < 0.05) were considered significant. If GLM tests indicated a significant difference between means, subsequent Tukey's multiple range tests was done to determine which of the means differed from each other. Main effect and differences were considered significant when $P<0.05$.

\section{RESULTS}

\section{Chemical Composition and Biomass Degradation of Rice Straw and Alfalfa Hay}

The chemical composition of the individual forages is listed in Supplemental Table S1 (http://dx.doi. org/10.3168/jds.2016-11398). Compared with rice straw, alfalfa hay had greater amounts of $\mathrm{CP}$ and ether extract, and lower amounts of NDF, ADF, and ash. During the rumen incubation period, alfalfa hay had higher DM loss $(P<0.001)$, NDF degradation $(P=$ $0.001)$, and $\mathrm{CP}$ degradation $(P<0.001)$ than rice straw (Supplemental Table S2; http://dx.doi.org/10.3168/ jds.2016-11398). Dry matter loss, NDF degradation, and CP degradation were significantly affected by incubation time $(P<0.001$, Supplemental Table S2).

\section{Data Acquisition and Analysis}

In this study, a 16S rRNA gene sequence analysis of samples generated 1,865,166 sequences with a length greater than 400 bp (Supplemental Table S3; http:// dx.doi.org/10.3168/jds.2016-11398) and an average of $51,810 \pm 6,115$ sequences per sample. Quality filtering by QIIME generated 1,230,669 high-quality reads, accounting for $66 \%$ of the raw reads, and an average of $34,185 \pm 8,724$ sequences per sample. The average sequence length was $437 \mathrm{bp}$.

\section{Dominant Taxa Colonizing the Rice Straw and Alfalfa Hay at $\mathbf{O}$ and Their Fate During Rumen Incubation}

The microbial communities colonizing the nonincubated $(0 \mathrm{~h})$ rice straw and alfalfa were both dominated by the members of Proteobacteria (70.47\%), Actinobacteria (18.69\%), Bacteroidetes (6.30\%), and Firmicutes (4.26\%) (Supplemental Table S4; http:// dx.doi.org/10.3168/jds.2016-11398). The $16 \mathrm{~S}$ rRNA data showed that the relative abundances of the communities colonizing the rice straw and alfalfa both decreased substantially to very low levels within the $0.5 \mathrm{~h}$ of rumen incubation, with some taxa decreas- ing to below detection limits (Supplemental Figure S1; http://dx.doi.org/10.3168/jds.2016-11398).

\section{Diversity of the Bacterial Microbiota Attached to Rice Straw and Alfalfa After 0.5 h of Rumen Incubation}

We assessed whether sampling provided sufficient OTU coverage to describe the bacterial composition of each region accurately by generating individually based rarefaction curves for each sample (Supplemental Figure S2; http://dx.doi.org/10.3168/jds.2016-11398). The Good's coverage was greater than 0.98, implying that sampling was sufficient for the samples collected at all incubation times (Supplemental Table S3; http:// dx.doi.org/10.3168/jds.2016-11398). At the 0.03 dissimilarity level, the OTU numbers and the abundancebased coverage estimator (ACE), Chao 1, and Shannon indices were significantly affected $(P<0.001$ to $P$ $=0.005)$ by the forage types and incubation time. The ACE, Chao 1, and Shannon indices were lower in both the rice straw and alfalfa samples collected at $48 \mathrm{~h}$ of incubation compared with 30 min of incubation (Table $1)$.

Principal coordinates analysis plots based on unweighted UniFrac distance metrics revealed that the alfalfa-colonizing microbiota exhibited clearly noticeable changes that paralleled the changes in incubation time (AMOVA: $F$-statistic $=1.91, P=0.01)$ (Figure 1). By contrast, the microbiota attached to the rice straw were not markedly different from each other (AMOVA: $F$-statistic $=0.97, P=0.513$ ). Analysis of molecular variance also showed that the ruminal bacterial communities attached to the rice straw (AMOVA: $F$-statistic $=2.85, P=0.006)$ were significantly different from the colonizing microbiota of the alfalfa.

Hierarchical cluster analysis of the dendrogram showed that 2 clusters were formed, and it also revealed differences in the composition of microbiota between rumen incubated samples (cluster 1) and nonincubated samples (cluster 2) (Supplemental Figure S3; http:// dx.doi.org/10.3168/jds.2016-11398). Cluster 2 included 2 distinct subclusters. Most samples from 16 and $48 \mathrm{~h}$ of incubation fell within subcluster 1 . Conversely, subcluster 2 primarily comprised samples from $0.5,2$, and $6 \mathrm{~h}$ of incubation.

\section{Characterization and Comparison of the Bacterial Communities Attached to Rice Straw and Alfalfa After 0.5 h of Rumen Incubation}

A total of 15 bacterial phyla were identified in all samples (Supplemental Figure S4; http://dx.doi. org/10.3168/jds.2016-11398). The majority of the se- 


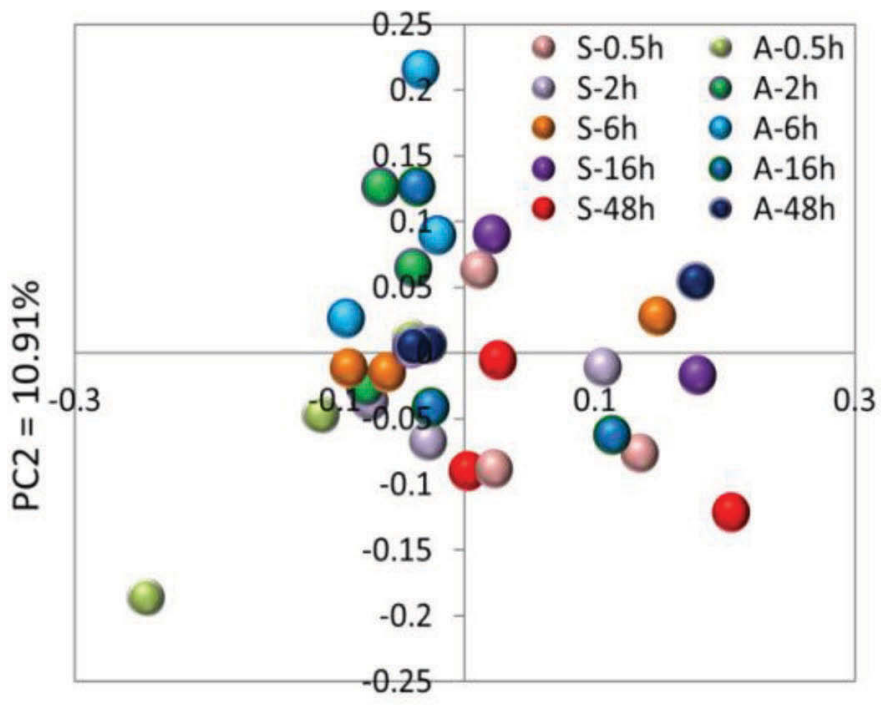

Figure 1. Principal coordinate analysis (PCoA) of microbial diversity across all samples using a unweighted UniFrac metric. The percentage of variation explained by $\mathrm{PC} 1$ and $\mathrm{PC} 2$ are indicated in the axis. $\mathrm{S}=$ rice straw samples; $\mathrm{A}=$ alfalfa samples. Color version available online.

quences obtained from the samples of rumen-incubated rice straw and alfalfa hay belonged to Firmicutes (rice straw, $34.19 \%$ vs. alfalfa, 39.05\%), Bacteroidetes (rice straw, $52.84 \%$ vs. alfalfa, 50.91\%), Spirochaetae (rice straw, $2.47 \%$ vs. alfalfa, $1.83 \%$ ), Tenericutes (rice straw, $2.17 \%$ vs. alfalfa, $2.81 \%$ ), Proteobacteria (rice straw, $2.08 \%$ vs. alfalfa, $1.56 \%$ ), and Fibrobacteres (rice straw, $2.01 \%$ vs. alfalfa, $0.42 \%$ ). The other 9 phyla were relatively minor $(<2 \%$ of total sequences) in abundance in comparison.
At the genus level, 159 and 163 taxa were observed across all rumen-incubated rice straw and alfalfa samples. For clarity and visualization purposes, the top 50 most abundant taxa detected in the present study are presented in Supplemental Figure S5 (http://dx.doi. org/10.3168/jds.2016-11398). The most abundant taxa (those with a relative abundance of $\geq 3 \%$ for at least 1 incubation time) attached to the rumen-incubated rice straw and alfalfa hay samples included Fibrobacter, Prevotella, Ruminococcus, Treponema, Butyrivibrio, and Anaeroplasma, as well as the unclassified taxa derived from Bacteroidales (family), Christensenellaceae (family), Lachnospiraceae (family), Prevotellaceae (family), Rikenellaceae (order), and Ruminococcaceae (family). On a genus level, the abundance of the dominant taxa Anaeroplasma, Butyrivibrio, Fibrobacter, Prevotella, unclassified Bacteroidales, unclassified Rikenellaceae, and unclassified Ruminococcaceae were affected (FDR < 0.001 to FDR $=0.028$ ) by the forage diet (Table 2), and the percentage of Anaeroplasma, Butyrivibrio, Fibrobacter, Prevotella, Ruminococcus, Treponema, unclassified Bacteroidales, unclassified Christensenellaceae, unclassified Lachnospiraceae, unclassified Rikenellaceae, and unclassified Ruminococcaceae changed (FDR $<0.001$ to $\mathrm{FDR}=0.048)$ over time.

The samples of rumen-incubated rice straw showed that most of the dominant taxa Butyrivibrio, Prevotella, and unclassified Christensenellaceae decreased after $0.5 \mathrm{~h}$ of incubation $(P<0.05)$ (Table 2), while Treponema, unclassified Bacteroidales, and unclassified Rikenellaceae increased in abundance at $48 \mathrm{~h}$ of incubation compared with the levels at $0.5 \mathrm{~h}$, with the highest amounts occurring in the samples at $48 \mathrm{~h}$ of incubation. A higher abundance of Fibrobacteria was observed

Table 1. The $\alpha$ diversity of bacterial community attached to rice straw and alfalfa hay at different incubation times

\begin{tabular}{|c|c|c|c|c|c|c|c|c|}
\hline \multirow[b]{2}{*}{ Item } & \multicolumn{2}{|c|}{ OTU $^{1}$ numbers } & \multicolumn{2}{|c|}{$\mathrm{ACE}^{2}$} & \multicolumn{2}{|c|}{ Chao 1 value } & \multicolumn{2}{|c|}{ Shannon index } \\
\hline & Rice straw & Alfalfa & Rice straw & Alfalfa & Rice straw & Alfalfa & Rice straw & Alfalfa \\
\hline $2 \mathrm{~h}$ & $1,578^{\mathrm{a}}$ & $1,649^{\mathrm{b}}$ & $1,761^{\mathrm{ab}}$ & $1,802^{\mathrm{a}}$ & $1,770^{\mathrm{ab}}$ & $1,820^{\mathrm{a}}$ & $6.07^{\mathrm{a}}$ & $6.12^{\mathrm{ab}}$ \\
\hline $6 \mathrm{~h}$ & $1,402^{\mathrm{a}}$ & $1,580^{\mathrm{b}}$ & $1,638^{\mathrm{ab}}$ & $1,740^{\mathrm{a}}$ & $1,658^{\mathrm{ab}}$ & $1,759^{\mathrm{a}}$ & $5.89^{\mathrm{ab}}$ & $6.05^{\mathrm{ab}}$ \\
\hline $16 \mathrm{~h}$ & $1,513^{\mathrm{a}}$ & $1,588^{\mathrm{b}}$ & $1,695^{\mathrm{ab}}$ & $1,763^{\mathrm{a}}$ & $1,721^{\mathrm{ab}}$ & $1,786^{\mathrm{a}}$ & $6.04^{\mathrm{a}}$ & $6.10^{\mathrm{ab}}$ \\
\hline $48 \mathrm{~h}$ & $1,377^{\mathrm{b}}$ & $1,388^{\mathrm{b}}$ & $1,576^{\mathrm{b}}$ & $1,602^{\mathrm{b}}$ & $1,602^{\mathrm{b}}$ & $1,628^{\mathrm{b}}$ & $5.50^{\mathrm{b}}$ & $5.63^{\mathrm{b}}$ \\
\hline $\mathrm{F}$ & \multicolumn{2}{|c|}{$<0.001$} & \multicolumn{2}{|c|}{0.01} & \multicolumn{2}{|c|}{0.005} & \multicolumn{2}{|c|}{$<0.001$} \\
\hline $\mathrm{T}$ & \multicolumn{2}{|c|}{$<0.001$} & \multicolumn{2}{|c|}{$<0.001$} & \multicolumn{2}{|c|}{$<0.001$} & \multicolumn{2}{|c|}{$<0.001$} \\
\hline $\mathrm{F} \times \mathrm{T}$ & \multicolumn{2}{|c|}{0.219} & \multicolumn{2}{|c|}{0.916} & \multicolumn{2}{|c|}{0.915} & \multicolumn{2}{|c|}{$<0.001$} \\
\hline
\end{tabular}

\footnotetext{
a,b Values within the same column with different superscripts were significantly different $(P<0.05)$.

${ }^{1} \mathrm{OTU}=$ operational taxonomic units.

${ }^{2} \mathrm{ACE}=$ abundance-based coverage estimator.

${ }^{3} \mathrm{SEM}=$ standard error of the difference of the means.

${ }^{4} \mathrm{~F}=$ effect of forage types; $\mathrm{T}=$ effect of incubation time; $\mathrm{F} \times \mathrm{T}=$ interaction effect of forage types and incubation time.
} 


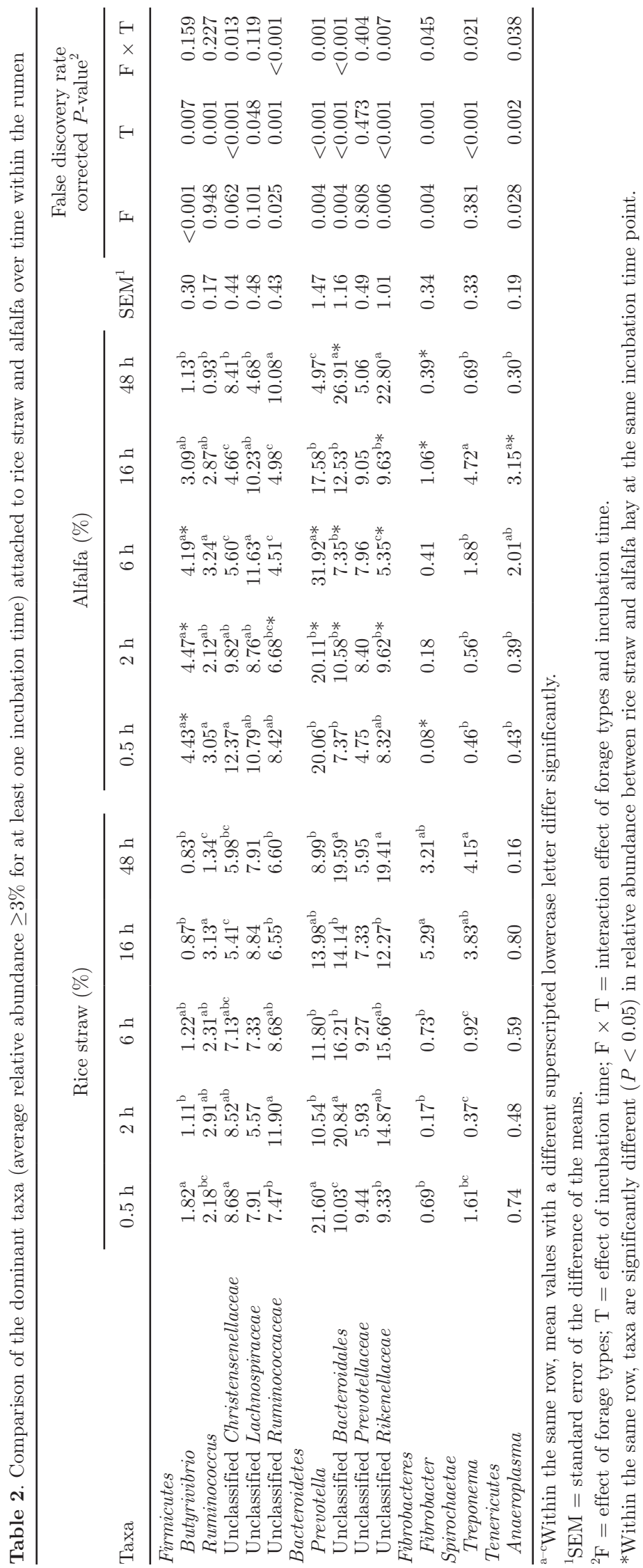


at $16 \mathrm{~h}$ of incubation compared with $0.5,2$, and $6 \mathrm{~h}$ of incubation. The proportion of genus Ruminococcus was higher at 2,6 , and $16 \mathrm{~h}$ of incubation compared with $48 \mathrm{~h}(P<0.05)$ (Table 2$)$. The samples of rumenincubated alfalfa showed no change in the proportion of Butyrivibrio $(P>0.05)$ from 0.5 to $16 \mathrm{~h}$ of incubation and a significant decrease $(P<0.05)$ at $48 \mathrm{~h}$ of incubation. The proportion of Prevotella was higher at $6 \mathrm{~h}$ of incubation compared with those at $0.5,2,16$, and 48 h. Higher percentages of Treponema and Anaeroplasma were observed at $16 \mathrm{~h}$ of incubation compared with those at $0.5,2$, and $48 \mathrm{~h}$. The unclassified Bacteroidales and unclassified Rikenellaceae increased in abundance after $0.5 \mathrm{~h}$ of incubation, with the greatest abundance at $48 \mathrm{~h}$. The abundances of Ruminococcus and unclassified Lachnospiraceae were unchanged from 2 to $16 \mathrm{~h}$ of incubation. The percentage of unclassified Ruminococcaceae was lower at 6 and $16 \mathrm{~h}$ of incubation than at 0.5 and $48 \mathrm{~h}$.

At the genus level, the proportion of Butyrivibrio was significantly higher in alfalfa samples than in rice straw samples at $0.5,2$, and $6 \mathrm{~h}$ of incubation, and the rice straw presented a higher $(P<0.05)$ percentage of unclassified Ruminococcaceae at $2 \mathrm{~h}$ of incubation (Table 2). At 2 and $6 \mathrm{~h}$ of incubation, a higher percentage of Prevotella was observed $(P<0.05)$ in alfalfa samples compared with the rice straw, while unclassified Rikenellaceae and unclassified Bacteroidales were more abundant $(P<0.05)$ in the rice straw samples. The abundance of Fibrobacter was significantly higher in rice straw samples than in alfalfa samples at 16 and $48 \mathrm{~h}$ of incubation, and rice straw samples also presented a higher $(P<0.05)$ percentage of Treponema at $0.5 \mathrm{~h}$ of incubation. Anaeroplasma were more abundant $(P<0.05)$ in the alfalfa samples compared with the rice straw samples at $16 \mathrm{~h}$ of incubation. Alfalfa samples presented a higher abundance of unclassified Bacteroidales compared with rice straw samples at 48 $\mathrm{h}$ of incubation.

\section{Predicted Functions of Bacteria Attached to Rice Straw and Alfalfa Hay After $0.5 \mathrm{~h}$ of Rumen Incubation}

Using PICRUSt as a predictive exploratory tool, we found that a total of 40 gene families occurred in all samples (Figure S6; http://dx.doi.org/10.3168/ jds.2016-11398). We performed PLS-DA on the relative abundance values of KEGG pathways represented from the bacteria associated with rice straw and alfalfa and found a clear distinction between the clustering of rice straw and alfalfa hay samples (Figure 2-A). Of the 40 gene families, the majority of the genes that were ob- tained belonged to replication and repair ( $10.76 \%$ of total genes inferred by PICRUSt), amino acid metabolism $(10.25 \%)$, membrane transport $(9.87 \%)$, carbohydrate metabolism (10.22\%), energy metabolism $(6.11 \%)$, and translation $(6.30 \%)$. Of the 40 gene families, 21 were significantly affected $(\mathrm{FDR}<0.05)$ by the forage types (Table S5; http://dx.doi.org/10.3168/jds.2016-11398), and 33 were significantly changed $(\mathrm{FDR}<0.05)$ over time. Of the 6 predominant gene families mentioned earlier, in the rumen-incubated rice straw samples, the relative abundances of the genes involved in carbohydrate metabolism decreased significantly from 0.5 to $48 \mathrm{~h}$ of incubation, while the abundance of genes involved in amino acid metabolism increased significantly $(P<0.05)$ after incubation (Figure $2 \mathrm{~B})$. In the rumen-incubated alfalfa samples, the proportion of the gene families related to energy metabolism, replication and repair, amino acid metabolism, and translation increased significantly $(P<0.05)$ from 0.5 to $48 \mathrm{~h}$ of incubation, while the abundance of genes involved in membrane transport decreased significantly after incubation, and no significant difference $(P>0.05)$ was observed in the proportion of the gene families related to carbohydrate metabolism within $48 \mathrm{~h}$ of incubation.

\section{Attached Bacterial Density}

Total bacterial populations in the rice straw and alfalfa hay samples were estimated with a real-time PCR analysis by measuring the total copy number of bacterial $16 \mathrm{~S}$ rRNA genes. The forage quality and incubation time both had a significant effect on the copy number of bacterial 16S rRNA genes determined on rice straw and alfalfa hay (Table 3). The highest bacterial number in the samples of rumen-incubated rice straw was observed at $48 \mathrm{~h}$ of incubation. The alfalfa-associated bacterial densities increased after incubation, and the greatest bacterial numbers were also observed at $48 \mathrm{~h}$ of incubation. Higher amounts of attached bacterial $16 \mathrm{~S}$ rRNA $(P<0.05)$ were observed in the alfalfa hay samples than in the rice straw from 0.5 to $16 \mathrm{~h}$ of incubation.

\section{Biomass Degradation Coordinated with Microbial Colonization}

Dry matter and CP degradation of rice straw and alfalfa hay was rapidly initiated within $0.5 \mathrm{~h}$ of rumen incubation (Figure 3 and Table S2; http://dx.doi. org/10.3168/jds.2016-11398). Corresponding to this period, we observed noticeable increases in Prevotella, Ruminococcus, unclassified Bacteroidales, unclassified Rikenellaceae, unclassified Lachnospiraceae, unclassi- 
A

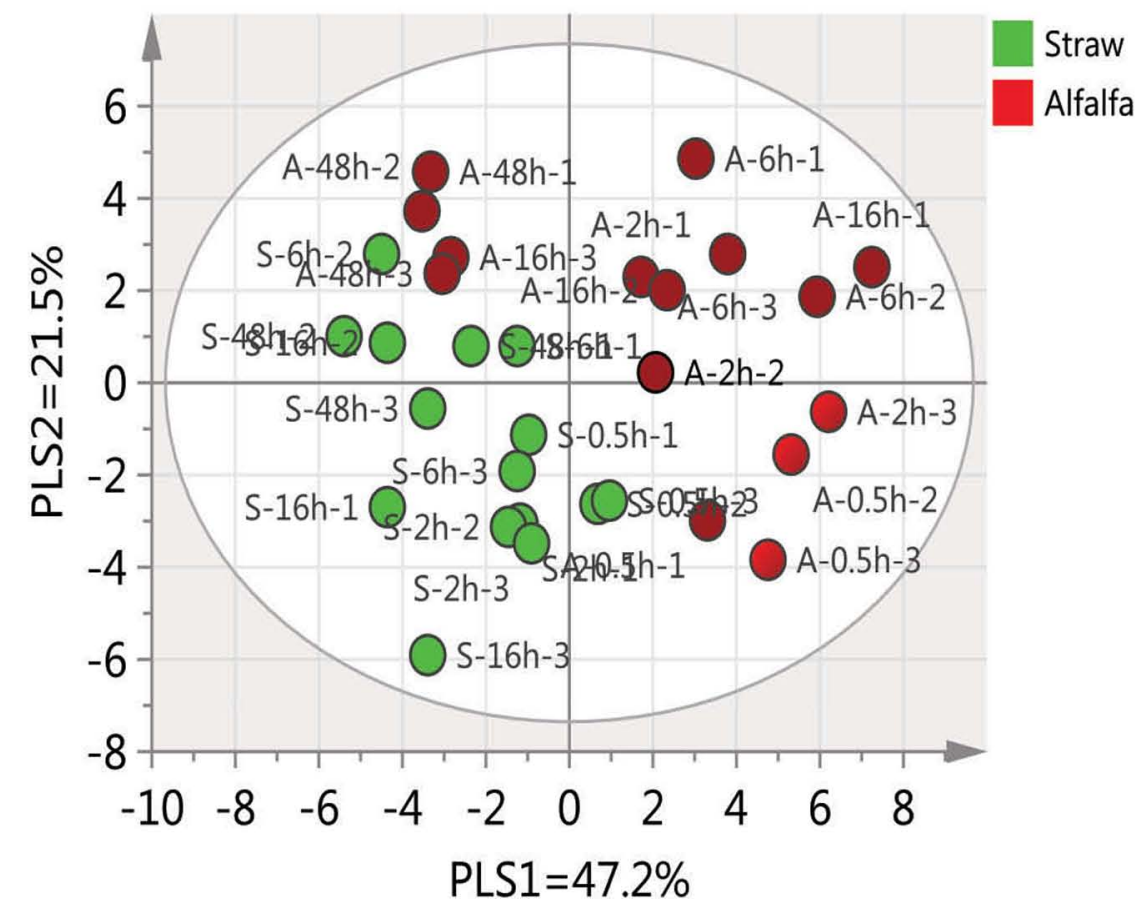

$\mathrm{B}$

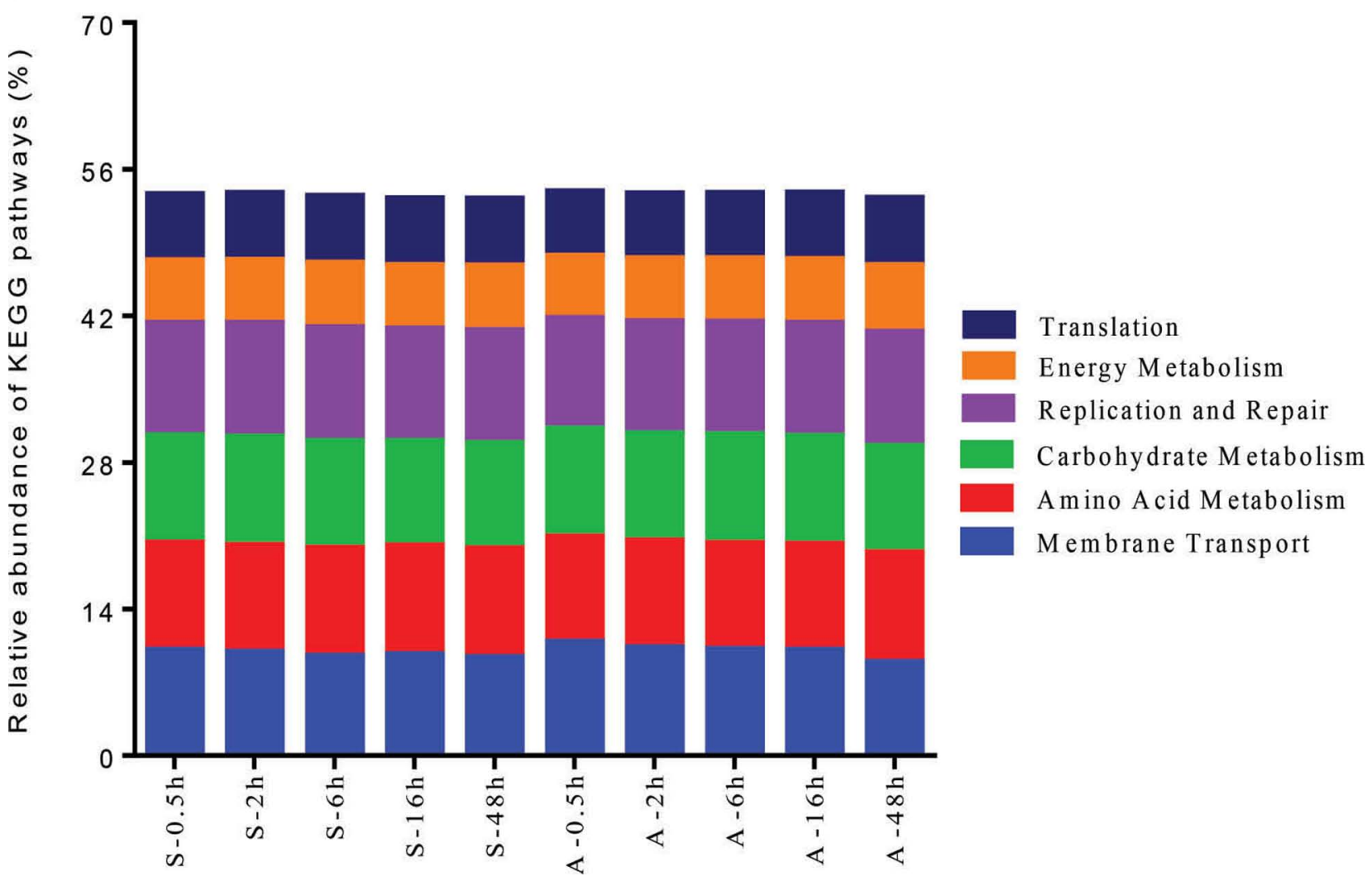

Figure 2. Metagenomic functional predictions for the samples. (A) Partial least squares (PLS) discriminant analysis of microbial functional diversity across all incubated samples using the relative abundances of functional pathways. (B) Comparisons of the 5 predominant gene pathways of the bacterial microbiota detected in the incubated sample. $\mathrm{S}=$ rice straw samples; $\mathrm{A}=$ alfalfa samples; KEGG = Kyoto Encyclopedia of Genes and Genomes. Color version available online. 
Table 3. Total bacterial numbers ( $\log _{10}$ gene copies/g of undigested forage) attached to rice straw and alfalfa hay following incubation for up to $48 \mathrm{~h}$

\begin{tabular}{|c|c|c|c|c|c|c|c|c|c|}
\hline \multirow[b]{2}{*}{ Forage } & \multicolumn{5}{|c|}{ Incubation time } & \multirow[b]{2}{*}{$\mathrm{SEM}^{1}$} & \multicolumn{3}{|c|}{$P$-value ${ }^{2}$} \\
\hline & $0.5 \mathrm{~h}$ & $2 \mathrm{~h}$ & $6 \mathrm{~h}$ & $16 \mathrm{~h}$ & $48 \mathrm{~h}$ & & $\mathrm{~F}$ & $\mathrm{~T}$ & $\mathrm{~F} \times \mathrm{T}$ \\
\hline $\begin{array}{l}\text { Rice straw } \\
\text { Alfalfa }\end{array}$ & $\begin{array}{l}10.40^{\mathrm{d}} \\
10.60^{\mathrm{b}}\end{array}$ & $\begin{array}{c}8.12^{\mathrm{e}} \\
10.86^{\mathrm{b} *}\end{array}$ & $\begin{array}{l}10.77^{\mathrm{c}} \\
11.15^{\mathrm{a} *}\end{array}$ & $\begin{array}{l}11.02^{\mathrm{b}} \\
11.22^{\mathrm{a} *}\end{array}$ & $\begin{array}{l}11.18^{\mathrm{a}} \\
11.12^{\mathrm{a}}\end{array}$ & 0.12 & $<0.001$ & $<0.001$ & $<0.001$ \\
\hline
\end{tabular}

${ }^{a-e}$ Mean values with different superscripted lowercase letters within the same row differ significantly $(P<0.05)$.

${ }^{1} \mathrm{SEM}=$ standard error of the difference of the means.

${ }^{2}$ Probability of a significant effect due to forage types $(\mathrm{F})$, incubation time $(\mathrm{T})$, and their interaction $(\mathrm{F} \times \mathrm{T})$.

*Within the same column, means differ significantly $(P<0.05)$.

fied Christensenellaceae, unclassified Prevotellaceae, and unclassified Ruminococcaceae from 0 to $0.5 \mathrm{~h}$ of incubation. From 0.5 to $48 \mathrm{~h}$ of incubation, DM degradation of rice straw proceeded somewhat linearly, at a rate of $\sim 0.60 \%$ biomass per hour. The total biomass degradation of alfalfa hay from 0.5 to $16 \mathrm{~h}$ of incubation occurred at a rate of $1.87 \%$ biomass per hour of incubation, and it decreased to $0.23 \%$ biomass per hour of incubation following $16 \mathrm{~h}$ of incubation. We observed noticeable decreases in the abundance of Fibrobacter, Treponema, and Butyrivibrio that corresponded to this period. Compared with the rice straw, the degradation of $\mathrm{CP}$ and NDF in alfalfa hay following rumen incubation appeared to be triphasic, and the degradation of $\mathrm{CP}$ and NDF degradation mainly occurred between 6 and $16 \mathrm{~h}$, corresponding to degradation of 20 and $40 \%$ of the total biomass and an increased abundance of taxa assigned to unclassified Rikenellaceae and Treponema species in the microbial communities.

We evaluated the effect of the content of the feed ingredients on the composition of microbiota colonizing the rice straw and alfalfa by performing a redundancy analysis using NDF, CP, and neutral detergent-soluble non-crude protein (DM - NDF - CP) as constrained explanatory variables and the relative abundances of dominant genera (those with a relative abundance of $\geq 2 \%$ for at least 1 incubation time) as response variables (Figure 4). Redundancy analysis of the composition of the microbial communities identified the NDF (pseudo$F=10.2, P=0.002$ ), CP (pseudo- $F=5.2, P=0.002$ ), and neutral detergent-soluble non-crude protein (pseudo- $F=2.6, P=0.036$ ) as significant factors, explaining 27.3 (Axis 1) and $11.1 \%$ (Axis 2), respectively, of the variation in microbial composition (Figure 4). On the redundancy analysis ordination plot, 10 genera, including Butyrivibrio, Anaeroplasma, Pseudobutyrivibrio, Succiniclasticum, Prevotella, Ruminococcus, Saccharofermentans, unclassified Lachnospiraceae, unclassified Christensenellaceae, and unclassified Lentisphaerae, were correlated with the $\mathrm{CP}$ content, while Bacteroi- des, Saccharofermentans, Ruminococcus, Papillibacter, Succiniclasticum, Prevotella, Fibrobacter, unclassified Ruminococcaceae, unclassified Lentisphaerae, unclassified Prevotellaceae, unclassified Mollicutes, unclassified Bacteria, and unclassified Christensenellaceae were related to the $\mathrm{CP}$.

\section{DISCUSSION}

We evaluated the temporal changes in the ruminal bacterial community colonizing and degrading rice straw and alfalfa hay in dairy cattle. In the present study, our MiSeq sequencing of $16 \mathrm{~S}$ rRNA revealed substantial temporal changes occurring within the first 30 min and after $6 \mathrm{~h}$ of rumen incubation. The major shift in the community composition during the first $30 \mathrm{~min}$ was phylum Proteobacteria being replaced by members belonging to the Bacteroidetes and Firmicutes. The change to a secondary phase was mainly associated with decreases in abundance of the taxa Butyrivibrio, Prevotella, Ruminococcus, and unclassified Christensenellaceae and increases in Fibrobacter, Treponema, unclassified Bacteroidales, and unclassified Rikenellaceae. These observations are consistent with analogous trials on rumen-incubated air-dried switchgrass, for which pyrosequencing-based analyses identified discrete microbial profiles within the first $30 \mathrm{~min}$ and after 4 $\mathrm{h}$ of rumen incubation (Piao et al., 2015). Our data also revealed that the temporal changes in the adherent microbiota are associated with the biomass loss of rice straw and alfalfa hay; indeed, the rates of rice straw and alfalfa degradation were greatest within the first 30 min of rumen incubation during which 20.6 and $30.1 \%$ of the total biomass was lost, respectively, and this may reflect a rapid utilization of soluble sugars and other easily fermentable nutrients. On the other hand, the bacteria detected from 16 to $48 \mathrm{~h}$ play a more focused role in plant fiber degradation, which is reflected in the results showing that more fiber-degrading bacteria, such as Fibrobacter and Treponema, were observed in 
the samples of rice straw and that a higher proportion of unclassified Bacteroidales was present in alfalfa hay samples from 16 to $48 \mathrm{~h}$ of incubation than from 0.5 to $6 \mathrm{~h}$.
Limited information is available on the effects of different forage types on the structure, diversity, and density of the attached microbiota. In the present study, our results suggest that forage types strongly

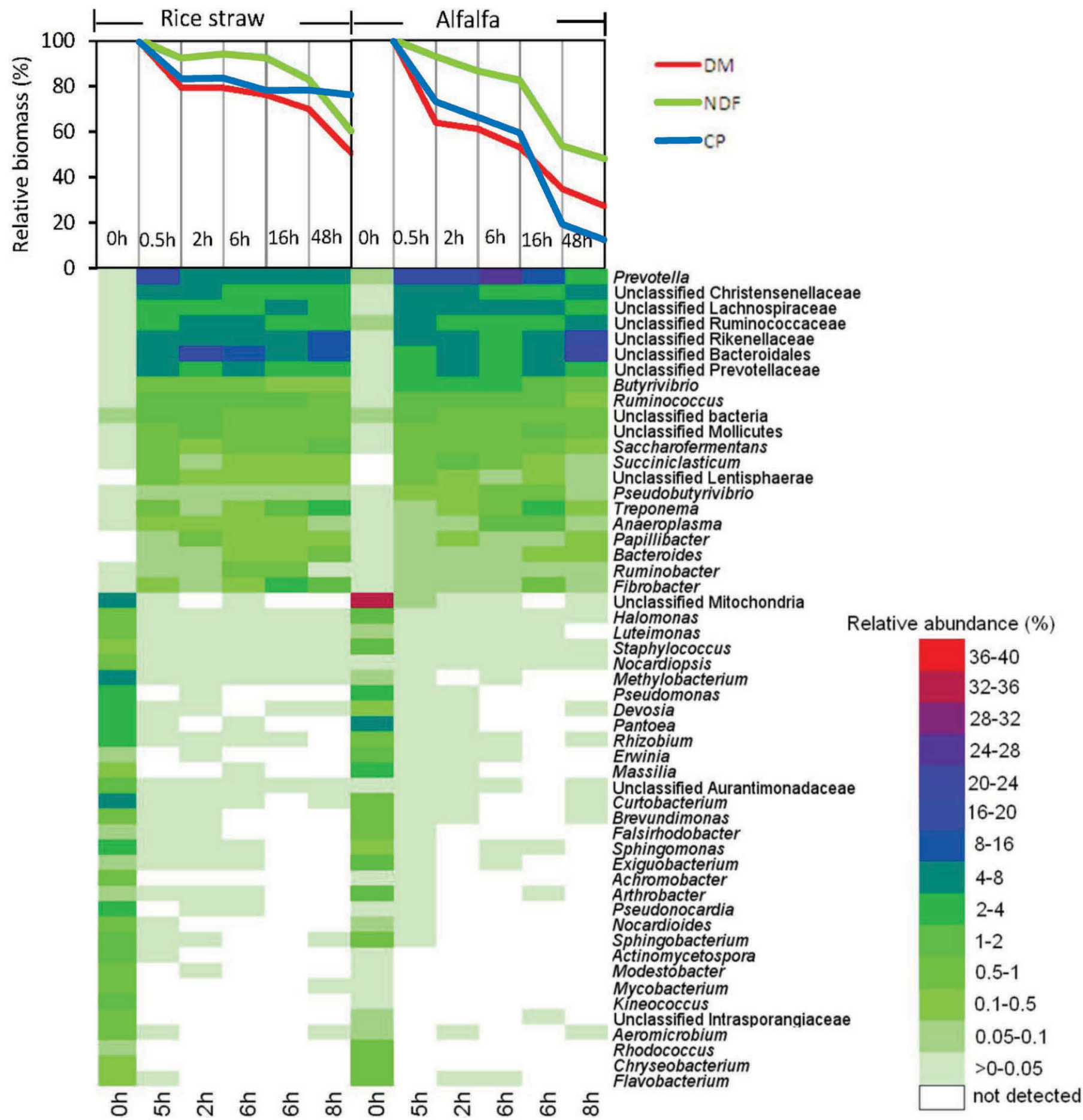

Figure 3. Microbial succession and biomass degradation during rumen incubation. Heat maps show succession of genera recruiting $>2 \%$ of the generated sequences. Line graphs show the relative DM change during rumen incubation. $\mathrm{S}=$ rice straw samples; A = alfalfa samples. Color version available online. 
affect the composition and structure of the attached microbiota of particles in the rumen of dairy cattle, as reflected by the clustering of the samples using PCoA and confirmed by AMOVA. Our findings also revealed that the 16S rRNA gene copy number of ruminal bacteria attached to the rice straw and alfalfa hay differed markedly from 0.5 to $16 \mathrm{~h}$ of incubation. We also found that more bacteria colonized the alfalfa hay than the rice straw, which may be partly explained by the differing physiochemical conditions, including the content of chemical ingredients and availability, between the 2 forages types. Indeed, our results showed that alfalfa hay had a greater content of neutral detergent solubles and a lower NDF content compared with rice straw. Thus, the difference in the composition and density of attached bacterial microbota between the 2 forage types is likely due to the differing chemical composition in the 2 forages, as mentioned earlier.

The present study revealed that the genera $\mathrm{Bu}$ tyrivibrio, Prevotella, Ruminococcus, Treponema, and Fibrobacter spp. dominated in both forages, and this finding is partly consistent with the report of Huws et al. (2016), who reported that Butyrivibrio, Fibrobacter, Olsenella, and Prevotella spp. abundances were high in the community of rumen bacteria colonizing fresh perennial ryegrass, indicating that these taxa play an important role in forage degradation in the rumen of dairy cattle. Of the dominant colonizing microbes detected in the present study, the abundances of Butyrivibrio, Fibrobacter, and Prevotella were significantly affected by the forage types. Butyrivibrio spp. are known to have proteolytic and polysaccharide-degrading activity (Fulghum and Moore, 1963; Kelly et al., 2010). In the present study, the RDA profile revealed that Butyrivibrio abundance was positively associated with the $\mathrm{CP}$ content. Thus, the fact that the abundance of Butyrivibrio was higher in alfalfa-associated microbiota than in the corresponding rice straw sample from 0.5 to $6 \mathrm{~h}$ of incubation might be partly explained by the higher content of $\mathrm{CP}$ in alfalfa hay compared with the rice straw. Prevotella has been reported as the most abundant genus in the rumen of adult dairy cattle, and it is

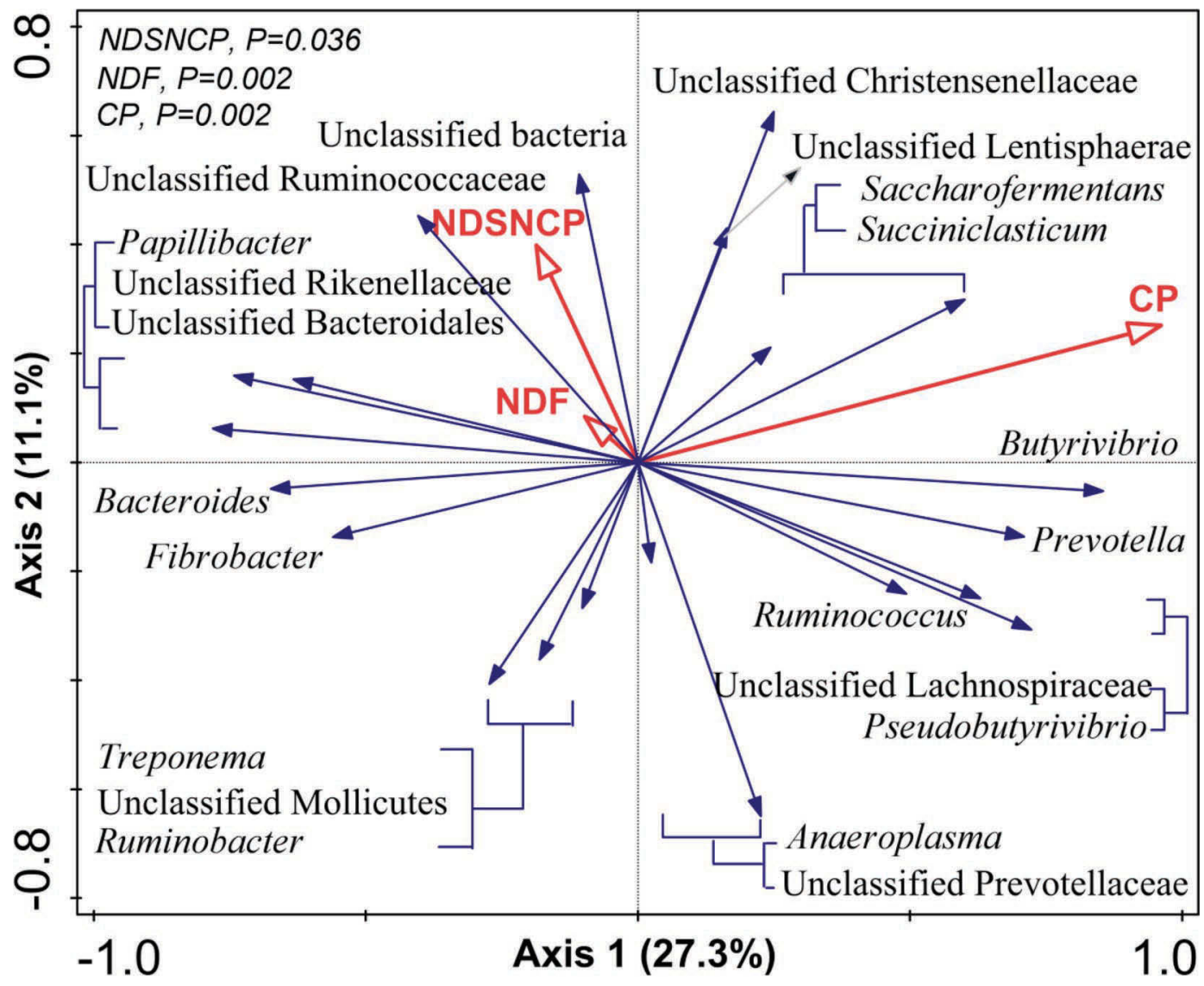

Figure 4. Biplot of the redundancy analysis (RDA) based on the relative abundance of dominant genera (>1\% of total sequence). Constrained explanatory variables are indicated by open triangle arrows. Neutral detergent-soluble non-crude protein (NDSNCP) is estimated as NDSNCP $=\mathrm{DM}-\mathrm{NDF}-\mathrm{CP}$. Upper left shows $P$-value of Monte Carlo permutation test. Color version available online. 
also associated with ruminal carbohydrate and protein fermentation (Wallace et al., 1999; Chiquette et al., 2008). The present study showed that Prevotella spp. dominated in the associated microbiomes of both rice straw and alfalfa, and this finding is consistent with a previous report (Stevenson and Weimer, 2007). In addition, the present study also revealed that the Prevotella abundance was positively associated with the content of $\mathrm{CP}$, and the content of $\mathrm{CP}$ is greater in the alfalfa hay compared with rice straw, providing a reasonable explanation for the higher abundance of Prevotella observed in the alfalfa samples than in the rice straw samples from 0.5 to $6 \mathrm{~h}$ of incubation. Fibrobacteria is one of the main cellulose-degrading bacterial genera in the rumen, and it may have an important role in the degradation of low-quality forages such as rice straw (Tajima et al., 2001). In the present study, correlation analysis revealed that Fibrobacteria abundance was positively associated with the content of NDF; thus, a higher abundance of Fibrobacteria was accordingly observed in the rice straw incubated for 16 and $48 \mathrm{~h}$ in the rumen than in the corresponding alfalfa hay samples.

The present study revealed that higher abundances of some unclassified groups, including unclassified Bacteroidales, unclassified Christensenellaceae, unclassified Lachnospiraceae, unclassified Prevotellaceae, unclassified Rikenellaceae, and unclassified Ruminococcaceae, were noted in particles of both forages. Among these organisms of unknown function, the Lachnospiraceae and Ruminococcaceae were the 2 largest groups of unclassified bacteria in the rumen. A meta-analysis revealed that these 2 unclassified bacterial groups are probably predominant ruminal bacteria and might have an important role in ruminal fiber digestion (Kim et al., 2011). However, little information is available regarding the possible function of the other unclassified groups, such as the Christensenellaceae and Prevotellaceae, in the rumen. In the present study, the reasons are not clear for why large abundances of unclassified groups were observed in the samples of rice straw and alfalfa hay and why some of these were affected by the incubation time and forage types. The explanations might be related to the potential roles of these organisms in the degradation of fiber and the dynamic changes in chemical composition of forage during incubation. Indeed, our previous study revealed that the unclassified Rikenellaceae and unclassified Ruminococcaceae dominated in the ruminal bacterial microbiota of dairy cattle fed a corn stover-based diet compared with an alfalfa-based diet (Zhang et al., 2014). In the present study, the RDA profiles showed that unclassified Rikenellaceae, unclassified Ruminococcaceae, and unclassified Bacteroidales were positively correlated with NDF content. Thus, the results indicate that these 3 unclassified populations may have an important role in the digestion of lowquality forages, such as rice straw.

We determined the potential functions of the attached bacteria in the samples by using PICRUSt to infer putative metagenomes from the $16 \mathrm{~S}$ rRNA gene profiles. The most abundant functional categories were those corresponding to the functions of amino acid metabolism, carbohydrate metabolism, membrane transport, and replication and repair. These findings are in agreement with general metabolic functions (such as carbohydrate, protein, and amino acid metabolism) being essential for microbial survival, and they are also consistent with the observations of other metagenomic studies in dairy cows (Parmar et al., 2014; Pitta et al., 2016). In addition, findings of the present study revealed significant differences in bacterial function depending on the incubation time. For example, genes relating to carbohydrate metabolism were more abundant in the rice straw-associated microbiota at $0.5,2$, and $6 \mathrm{~h}$ of incubation than at 16 and $24 \mathrm{~h}$, whereas the genes associated with amino acid metabolism were more enriched in the colonizing microbiota of both rice straw and alfalfa collected at $48 \mathrm{~h}$ of incubation than at $0.5 \mathrm{~h}$. In addition, the genes associated with carbohydrate metabolism were more enriched in the associated microbiota of alfalfa incubated from 6 to $48 \mathrm{~h}$ than in the corresponding rice straw digests. One possibility is that alfalfa provides a greater supply of easily fermentable carbohydrates during 6 to $48 \mathrm{~h}$ of incubation.

In summary, this study demonstrated that a highly diverse rumen bacterial community colonized rice straw and alfalfa hay rapidly and continuously changed during a 48-h incubation period, revealing high abundances of Butyrivibrio, Prevotella, Ruminococcus, Treponema, Fibrobacter and some unclassified microbes in the colonizing microbiome. The forage types and incubation times have a significant effect on the diversity, composition, structure, and potential function of colonizing microbiota, and RDA revealed that the content of $\mathrm{CP}$ and NDF have a significant effect on the composition of the attached microbiota. A greater abundance of Butyrivibrio, Prevotella, and unclassified Lachnospiraceae was observed in alfalfa hay samples, while the rice straw samples contained a higher proportion of Fibrobacter, Treponema, and unclassified Bacteroidales, indicating that the abundance of dominant taxa in colonizing microbiome is associated with the forage types. Generally, the results obtained here provide a comprehensive overview of the effects of 2 differing quality forages on the diversity, composition, structure, and temporal changes of colonizing microbiota, and they indicate that the rumen microbial colonization events are rapid within the 
rumen and that the differences in ecological community are dependent on the differing chemical composition, especially the contents of $\mathrm{CP}$ and NDF.

\section{CONCLUSIONS}

Collectively, our results reveal differing dynamics of bacterial colonization and inferred gene function of associated microbiota between the alfalfa hay and rice straw within the rumen. These findings are of great importance for the targeted improvement of forage nutrient use efficiency in ruminants.

\section{ACKNOWLEDGMENTS}

The present study was supported by the National Key Basic Research Program of China (2011CB100801).

\section{REFERENCES}

AOAC. 1990. Official Methods of Analysis. 16th ed. Association of Official Analytical Chemists, Arlington, VA.

Benjamini, Y., and Y. Hochberg. 2000. On the adaptive control of the false discovery rate in multiple testing with independent statistics. J. Educ. Behav. Stat. 25:60-83.

Bowman, J. G., and J. L. Firkins. 1993. Effects of forage species and particle size on bacterial cellulolytic activity and colonization in situ. J. Anim. Sci. 71:1623-1633.

Brask, M., P. Lund, A. L. F. Hellwing, M. Poulsen, and M. R. Weisbjerg. 2013. Enteric methane production, digestibility and rumen fermentation in dairy cows fed different forages with and without rapeseed fat supplementation. Anim. Feed Sci. Technol. 184:67-79.

Caporaso, J. G., K. Bittinger, F. D. Bushman, T. Z. DeSantis, G. L. Andersen, and R. Knight. 2010a. PyNAST: A flexible tool for aligning sequences to a template alignment. Bioinformatics $26: 266-267$.

Caporaso, J. G., J. Kuczynski, J. Stombaugh, K. Bittinger, F. D Bushman, E. K. Costello, N. Fierer, A. G. Pena, J. K. Goodrich, J. I. Gordon, G. A. Huttley, S. T. Kelley, D. Knights, J. E. Koenig, R. E. Ley, C. A. Lozupone, D. McDonald, B. D. Muegge, M. Pirrung, J. Reeder, J. R. Sevinsky, P. J. Tumbaugh, W. A. Walters, J. Widmann, T. Yatsunenko, J. Zaneveld, and R. Knight. 2010b. QIIME allows analysis of high-throughput community sequencing data. Nat. Methods 7:335-336.

Chiquette, J., M. J. Allison, and M. A. Rasmussen. 2008. Prevotella bryantii $25 \mathrm{~A}$ used as a probiotic in early-lactation dairy cows: Effect on ruminal fermentation characteristics, milk production, and milk composition. J. Dairy Sci. 91:3536-3543.

Craig, W. M., G. A. Broderick, and D. B. Ricker. 1987. Quantitation of microorganisms associated with the particulate phase of ruminal ingesta. J. Nutr. 117:56-62.

DeSantis, T. Z., P. Hugenholtz, N. Larsen, M. Rojas, E. L. Brodie, K. Keller, T. Huber, D. Dalevi, P. Hu, and G. L. Andersen. 2006. Greengenes, a chimera-checked 16S rRNA gene database and workbench compatible with ARB. Appl. Environ. Microbiol. 72:5069-5072

Ding, L. M., G. J. Lascano, and A. J. Heinrichs. 2015. Effect of precision feeding high- and low-quality forage with different rumen protein degradability levels on nutrient utilization by dairy heifers. J. Anim. Sci. 93:3066-3075.

Edgar, R. C. 2013. UPARSE: Highly accurate OTU sequences from microbial amplicon reads. Nat. Methods 10:996-998.

Edgar, R. C., B. J. Haas, J. C. Clemente, C. Quince, and R. Knight. 2011. UCHIME improves sensitivity and speed of chimera detection. Bioinformatics 27:2194-2200.
Edwards, J. E., S. A. Huws, E. J. Kim, and A. H. Kingston-Smith 2007. Characterization of the dynamics of initial bacterial colonization of nonconserved forage in the bovine rumen. FEMS Microbiol. Ecol. 62:323-335.

Fulghum, R. S., and W. E. Moore. 1963. Isolation, enumeration, and characteristics of proteolytic ruminal bacteria. J. Bacteriol $85: 808-815$.

Hook, S. E., M. A. Steele, K. S. Northwood, J. Dijkstra, J. France, A. D. G. Wright, and B. W. McBride. 2011. Impact of subacute ruminal acidosis (SARA) adaptation and recovery on the density and diversity of bacteria in the rumen of dairy cows. FEMS Microbiol. Ecol. 78:275-284.

Huws, S. A., J. E. Edwards, C. J. Creevey, P. Rees Stevens, W. Lin, S. E. Girdwood, J. A. Pachebat, and A. H. Kingston-Smith. 2016. Temporal dynamics of the metabolically active rumen bacteria colonizing fresh perennial ryegrass. FEMS Microbiol. Ecol. 92:fiv137. http://dx.doi.org/10.1093/femsec/fiv137.

Huws, S. A.. O. L. Mayorga, M. K. Theodorou, L. A. Onime, E. J. Kim, A. H. Cookson, C. J. Newbold, and A. H. Kingston-Smith. 2013. Successional colonization of perennial ryegrass by rumen bacteria. Lett. Appl. Microbiol. 56:186-196.

Kelly, W. J., S. C. Leahy, E. Altermann, C. J. Yeoman, J. C. Dunne, Z. H. Kong, D. M. Pacheco, D. Li, S. J. Noel, C. D. Moon, A. L. Cookson, and G. T. Attwood. 2010. The glycobiome of the rumen bacterium Butyrivibrio proteoclasticus B316(T) highlights adaptation to a polysaccharide-rich environment. PLOS ONE 5:e11942.

Kim, H., I. Lee, Y. Kwon, B. C. Kim, S. Ha, J. H. Lee, and J. Kim 2011. Immobilization of glucose oxidase into polyaniline nanofiber matrix for biofuel cell applications. Biosens. Bioelectron. 26:39083913

Langille, M. G., J. Zaneveld, J. G. Caporaso, D. McDonald, D. Knights, J. A. Reyes, J. C. Clemente, D. E. Burkepile, R. L. Vega Thurber, R. Knight, R. G. Beiko, and C. Huttenhower. 2013. Predictive functional profiling of microbial communities using $16 \mathrm{~S}$ rRNA marker gene sequences. Nat. Biotechnol. 31:814-821.

Larter, N. C. 1992. Forage fiber analyses-A comparison of 2 techniques. Wildl. Res. 19:289-293.

Lozupone, C., and R. Knight. 2005. UniFrac: A new phylogenetic method for comparing microbial communities. Appl. Environ. Microbiol. 71:8228-8235.

Mao, S., M. Zhang, J. Liu, and W. Zhu. 2015. Characterising the bacterial microbiota across the gastrointestinal tracts of dairy cattle: Membership and potential function. Sci. Rep. 5:16116.

Mao, S., R. Zhang, D. Wang, and W. Zhu. 2012. The diversity of the fecal bacterial community and its relationship with the concentration of volatile fatty acids in the feces during subacute rumen acidosis in dairy cows. BMC Vet. Res. 8:237.

McAllister, T. A., H. D. Bae, G. A. Jones, and K. J. Cheng. 1994 Microbial attachment and feed digestion in the rumen. J. Anim. Sci. 72:3004-3018.

Parmar, N. R., J. V. Solanki, A. B. Patel, T. M. Shah, A. K. Patel, S Parnerkar, J. I. N. Kumar, and C. G. Joshi. 2014. Metagenome of Mehsani buffalo rumen microbiota: An assessment of variation in feed-dependent phylogenetic and functional classification. J. Mol. Microbiol. Biotechnol. 24:249-261.

Piao, H., M. Lachman, S. Malfatti, A. Sczyrba, B. Knierim, M. Auer, S. G. Tringe, R. I. Mackie, C. J. Yeoman, and M. Hess. 2014 Temporal dynamics of fibrolytic and methanogenic rumen microorganisms during in situ incubation of switchgrass determined by 16S rRNA gene profiling. Front. Microbiol. 5:307.

Piao, H. L., E. Hawley, S. Kopf, R. DeScenzo, S. Sealock, T. HenickKling, and M. Hess. 2015. Insights into the bacterial community and its temporal succession during the fermentation of wine grapes. Front. Microbiol. 6:809

Pitta, D. W., N. Indugu, S. Kumar, B. Vecchiarelli, R. Sinha, L. D. Baker, B. Bhukya, and J. D. Ferguson. 2016. Metagenomic assessment of the functional potential of the rumen microbiome in Holstein dairy cows. Anaerobe 38:50-60.

Russell, J. B., and J. L. Rychlik. 2001. Factors that alter rumen microbial ecology. Science 292:1119-1122. 
Schloss, P. D., S. L. Westcott, T. Ryabin, J. R. Hall, M. Hartmann, E. B. Hollister, R. A. Lesniewski, B. B. Oakley, D. H. Parks, C. J. Robinson, J. W. Sahl, B. Stres, G. G. Thallinger, D. J. Van Horn, and C. F. Weber. 2009. Introducing mothur: open-source, platform-independent, community-supported software for describing and comparing microbial communities. Appl. Environ. Microbiol. 75:7537-7541.

Stevenson, D. M., and P. J. Weimer. 2007. Dominance of Prevotella and low abundance of classical ruminal bacterial species in the bovine rumen revealed by relative quantification real-time PCR. Appl. Microbiol. Biotechnol. 75:165-174.

Tajima, K., R. I. Aminov, T. Nagamine, H. Matsui, M. Nakamura, and Y. Benno. 2001. Diet-dependent shifts in the bacterial population of the rumen revealed with real-time PCR. Appl. Environ. Microbiol. 67:2766-2774.

Ter Braak, C. J., and P. Smilauer. 2012. Canoco Reference Manual and User's Guide: Software for Ordination. Version 5.0. Microcomputer Power, Ithaca, NY.
Wallace, R. J., C. Atasoglu, and C. J. Newbold. 1999. Role of peptides in rumen microbial metabolism-Review. Asian-australas. J. Anim. Sci. 12:139-147.

Wang, Q., G. M. Garrity, J. M. Tiedje, and J. R. Cole. 2007. Naive Bayesian classifier for rapid assignment of rRNA sequences into the new bacterial taxonomy. Appl. Environ. Microbiol. 73:5261-5267.

Welch, J. G., and A. M. Smith. 1969. Influence of forage quality on rumination time in sheep. J. Anim. Sci. 28:813-818.

Yang, H. E., Y. S. Son, and K. A. Beauchemin. 2011. Effects of exogenous enzymes on ruminal fermentation and degradability of alfalfa hay and rice straw. Asian-australas. J. Anim. Sci. 24:56-64.

Zhang, R., W. Zhu, W. Zhu, J. Liu, and S. Mao. 2014. Effect of dietary forage sources on rumen microbiota, rumen fermentation and biogenic amines in dairy cows. J. Sci. Food Agric. 94:1886-1895. 